\title{
A Proposal for a Standard Nomenclature and Interactive Database of Parameters and Correction Algorithms Used in Quantitative Electron Probe Microanalysis
}

\author{
*J. T. Armstrong, ${ }^{*}$ J. H. Scott, ${ }^{*}$ J. A. Small, and ${ }^{*}$ E. B. Steel \\ *Surface and Microanalysis Science Division, National Institute of Standards and Technology, \\ Gaithersburg, MD 20899-8371
}

Electron probe microanalysis (EPMA) is potentially one of the most accurate quantitative analysis methods - achieving relative accuracies of better than $1 \%$ under optimal conditions - and one of the most versatile techniques in terms of the range of analyzed elements and matrix complexity that can be handled without any pre-concentration or other types of specimen alteration. As the technique has matured over the past 50 years, it has become the standard method for non-destructive microanalysis in the geological and materials sciences as well as many other fields. Much work has been done to better understand the physical processes underlying the analytical technique and improve the correction procedures employed for quantitative analysis - so much work, in fact, that today literally dozens of different combinations of physical parameters and correction schemes are in use by the active laboratories involved in quantitative EPMA, and are contained in the software packages of the various commercial manufacturers of x-ray analysis instrumentation.

A certain sloppiness has settled into the EPMA community, in that exactly which parameters and algorithms have been employed to convert relative intensities to estimated elemental concentrations is seldom reported (nor typically are the raw k-ratios published). Generic correction titles like "ZAF procedures" or "Phi-Rho-Z methods" have become essentially meaningless because of the large number of variations that have been utilized. Sometimes different implementations of reputedly the same correction procedure yield significantly different results - either because of errors in the programming or undocumented changes/simplifications in equations and/or parameters. In some commercial systems, the correction algorithms and physical parameters contained in the software are proprietary information and unknown to the user. As a result it has become impossible to directly compare analytical results from different laboratories without adding an uncertainty due to the lack of knowledge of how the data has been manipulated. Few laboratories and no current commercial systems make any attempt to quantify the magnitude of this uncertainty in their error analysis, although there is abundant evidence that it is often considerably larger than the reported uncertainties due to counting statistics (e.g., [1-4]). Fig. 1 and 2 illustrate a typical case. Fig. 1 shows the range of mass absorption coefficients (MAC) of x-rays in the 1-1.8 keV range by Ge from three different tabulations of MACs by NIST investigators as well as the only set of measured values published for this energy region. Fig. 2 shows the range of results of Si concentration in SiGe alloys that would be obtained using the three MAC tabulations along with various commonly used correction procedures. The range of variation in this case exceeds $20 \%$.

We propose development of a set of standard nomenclature, algorithms, parameter databases and reporting procedures for quantitative EPMA to assist in quality assurance and the evaluation of inter-laboratory results. We propose the establishment of a database maintained on a web page by the Surface and Microanalysis Science Division of NIST that would list the exact equations and parameters used by the authors of published correction procedures and provide a nomenclature to 
describe such procedures. The listings should include the k-ratios calculated for selected sample and standard compositions under defined analytical conditions and compositions calculated for selected k-ratios allowing quality control checks of programs using these corrections (employing modifications of the 'NISTZAF' and 'TRYZAF' programs currently available through NIST [1-4]). We would solicit updates of new or revised correction procedures.

Along with the listing of correction procedures with a standardized nomenclature, we propose augmenting existing efforts by NIST, D. Joy [5] and others in maintaining tables of measured values of the parameters used in EPMA corrections, such as mass absorption coefficients, mean ionization potentials, fluorescence yields, backscatter coefficients, and ionization cross sections. The experimental data could be annotated with uncertainty estimates and comparison with the various parameterizations of these factors used in correction procedures. Finally, this database could serve as a repository of primary measurements of well-characterized standards by multiple laboratories to enable evaluation and refinement of present and future EPMA correction procedures.

It is hoped that there will be sufficient interest in the microbeam analysis community for such a data repository that a multi-laboratory data collection and evaluation committee can be established. A successful program of this type could lead to helpful new quality assurance procedures (including possible new ASTM and ISO standards) as well as ultimately improving the accuracy of quantitative EPMA for difficult analytical systems.

\section{References}

[1] J. T. Armstrong, Microsc. Microanal. $\underline{7}$ (suppl. 2) (2001) 670.

[2] J. T. Armstrong, Microsc. Microanal. $\underline{5}$ (suppl. 2) (1999) 560.

[2] J. T. Armstrong, Microsc. Microanal. 4 (suppl. 2) (1998) 216.

[4] J. T. Armstrong, Microsc. Microanal. 4 (suppl. 2) (1998) 226.

[5] D. C. Joy, Mikrochim. Acta 138 (2002) 105.

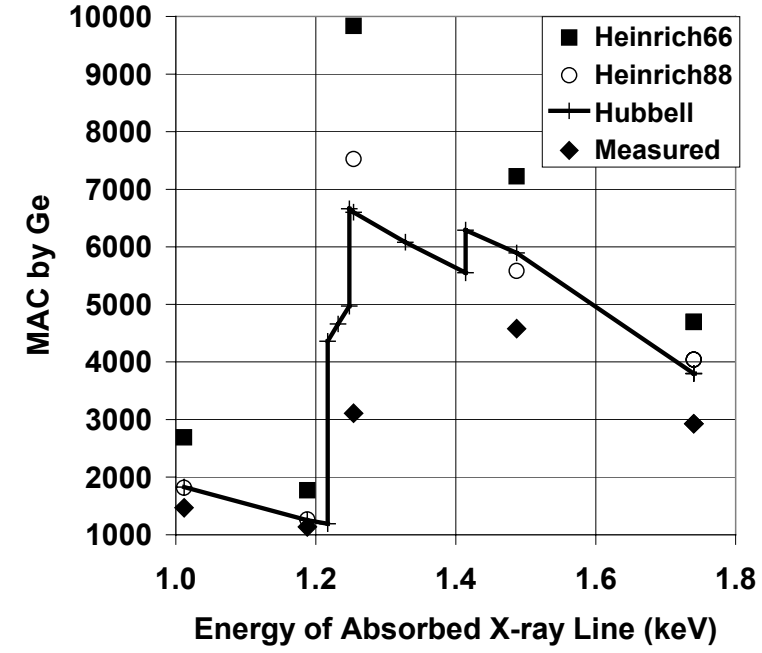

Figure 1. Plot of measured and tabulated mass absorption coefficients by Ge of x-ray lines with energies between 1 and $1.8 \mathrm{keV}$.
Si:Ge $20 \mathrm{keV}$ (Correction range)

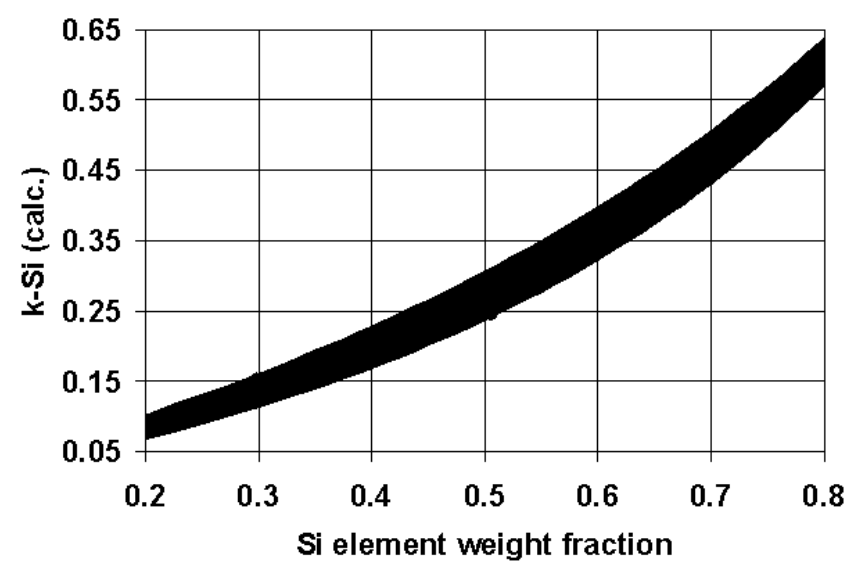

Figure 2. Range of calculated k-Si for Si:Ge alloys at $20 \mathrm{keV}$ using various correction procedures and mass absorption coefficients. 